\title{
El curriculum universitario y el enfoque de competencias
}

\author{
Marta Susana Brovelli \\ (Escuela de Ciencias de la Educación) \\ (Universidad Nacional de Rosario. Argentina) \\ (Universidad Tecnológica Nacional. Argentina)
}

\section{Resumen}

En este artículo se plantean los cambios en el contexto global: económico, político, social y cultural y, en consecuencia, los nuevos desafíos que debe enfrentar el curriculum universitario en este complejo escenario. En este marco se parte del Proceso de convergencia de Bologna y se pasa a analizar el concepto de competencias desde la perspectiva de diferentes autores, para centrarse luego en su irrupción en el campo de la educación y, muy especialmente en el campo del curriculum y de la didáctica.

Se trabaja el problema de la formación de competencias y la temporalidad, para plantear luego algunos riesgos y, también, algunas alternativas en la construcción del curriculum en el nivel superior.

El interrogante que está siempre presente es si el cambio curricular basado en competencias es percibido solamente como respuesta a demandas que provienen del exterior del campo educativo o si puede vivirse además como un cambio deseado y necesario o conveniente para mejorar la calidad de los profesionales que se forman en la universidad.

\section{Palabras clave}

Cambios - Desafíos - Competencias - Curriculum universitario - Alternativas.

\section{Summary}

This article presents changes in the global context of economic, political, social and cultural development and, consequently, new challenges faced by the university's curriculum in this complex scenario. Within this framework from the Bologna Process of Convergence, it comes the analysis of the concept of competences from the perspective of different authors, then focus on its entry into the field of education and especially in the field of curriculum and didactics.

The article also works the problem of skills training and temporality, to present later some risks and also some alternatives in the construction of the curriculum in the upper level. 
The question that is always present is whether the competency-based curriculum change is seen only in response to demands coming from outside the educational field or can be lived as well as a change desired or necessary or convenient to improve the quality of professionals formed in the university.

\section{Key words}

Changes - Challenges - Competences - University's curriculum - Alternatives.

\section{Introducción}

Los cambios macro políticos, económicos, sociales y culturales producidos en el mundo actual; los giros ideológicos impensados; el llamado proceso de globalización e internacionalización de la economía; los cambios respecto del papel del Estado han acarreado, como consecuencia, un reacomodamiento de los países en el concierto mundial de acuerdo con el lugar que pueden o aspiran a ocupar según sus posibilidades de crecimiento y competitividad, como así, también, según el tipo y grado de relación que mantengan con los nuevos centros de poder. Esto ha producido profundas reorganizaciones en las diferentes esferas de los gobiernos de los países, que han optado por distintas alternativas para afrontar la globalización.

La educación en general, y la universidad en particular, vienen sufriendo procesos de crítica acerca de sus funciones y de la calidad de las formaciones que logran. Estas críticas están especialmente originadas en los cambios profundos y variados que se han producido en los contextos sociales, políticos y económicos. Esta situación coloca a las instituciones educativas ante la exigencia de producir cambios en el curriculum y en la enseñanza con la intencionalidad de mejorar la calidad de las formaciones que pretenden lograr y de reformular sus políticas y acciones de funcionamiento interno y de articulaciones con otras instituciones educativas y sociales más amplias, como así también, con el mundo del trabajo profesional. Las instituciones educativas, que tienden naturalmente a ser conservadoras e impermeables a los cambios e innovaciones, se han visto sacudidas por estos fuertes cambios culturales y sociales, poniendo de manifiesto sus aspectos más anacrónicos y, entre ellos, muy especialmente las propuestas curriculares que ofrecen.

En cuanto a las relaciones entre la educación y los cambios socioeconómicos, pensamos que ellos no implican ineludiblemente una simple adaptación del sistema educativo a las necesidades económicas y sociales de un tiempo y espacio determinados. Esto es así, si se considera que la educación tiene funciones específicas que le son propias e irrenunciables, como las de la formación del ciudadano atendiendo a su crecimiento cultural, a la adquisición de nuevos conocimientos y al desarrollo de una capacidad crítica respecto 
del mundo en el que le toca vivir y trabajar. Sin embargo, las cambiantes características y exigencias del contexto sociopolítico y económico en el cual la educación tiene lugar, le plantean nuevas demandas o viejas demandas pero con nuevas respuestas.

Intentar ver qué sucede en el contexto actual con la educación, significa un difícil ejercicio crítico, que obliga a tratar de abordar una serie de tensiones y contradicciones que se están dando en este, de por sí, complejo ámbito. ¿Cómo pretender crecimiento, competitividad y reconocimiento en el mundo global desatendiendo la educación? ¿Cómo elevar la eficacia y calidad en este sector del Estado? ¿Cómo atender a una crisis educativa que ya no es coyuntural como se pensaba hace algunos años? ¿Cómo requerirle a la educación la preparación con eficacia de los ciudadanos críticos para enfrentar las exigencias del mundo en el que tienen y tendrán que vivir? ¿Qué tipo de relaciones pueden establecerse entre educación universitaria y mundo del trabajo?

Por otra parte ante el claro e irreversible contexto de globalización en que vivimos, ha surgido, por parte de los diferentes países, la necesidad y conveniencia de llegar a acuerdos en cuanto a formaciones y acreditaciones de estudios y profesiones, que faciliten el intercambio de estudiantes, profesores y profesionales, aspecto que está teniendo desde hace ya un tiempo considerable, clara importancia con aumento notorio de posibilidades. En tal sentido la construcción del Espacio Europeo de Educación Superior ("European Higher Educación Area" o "Bolonia process") en palabras de Bolívar (2007) (1), se ha constituido como una oportunidad histórica para armonizar la Educación Superior europea, ya que además de la señalada facilitación de intercambio este autor considera que puede facilitarse la producción de una renovación didáctica de la educación superior, incidiendo en el curriculum, el profesorado, la enseñanza, el trabajo de los estudiantes.

En Latinoamérica y el Caribe también existe preocupación por la organización y funcionamiento de la Educación Superior con claros avances en el campo, pudiéndose citar en tal sentido la reunión de Cartagena de Indias (2008) y la producción constante de trabajos y publicaciones al respecto.

Las situaciones mencionadas han ido creando las condiciones necesarias para pensar y diseñar acciones tendientes a la innovación educativa en general y, más específicamente, en el Nivel Superior. El curriculum se ha convertido en un objeto privilegiado para producir cambios, en tanto es nada más ni nada menos que el trayecto a seguir en la formación de los futuros profesionales en una diversidad cada vez más amplia.

Así es como este contexto favorable a la innovación curricular y de la enseñanza se ve atravesado por el impacto en el campo educativo de un nuevo (o no tan nuevo) concepto que en realidad proviene de otro campo como lo es el de competencias. Es así como el discurso de las "competencias" es introducido de manera acrítica pretendiendo que se convierta en modelo base para 
las nuevas formulaciones del diseño curricular (Bolívar, 2007). Díaz Barriga (2006, p. 29) (2) sostiene que el discurso se ha introducido sin analizar o desconociendo las bases conceptuales que subyacen a este tema y va también a argumentar que no puede convertirse en una metodología para la elaboración de diseños curriculares.

Es posible advertir que el discurso de las competencias en relación con los diseños curriculares de la Educación Superior, por un lado, ha tenido y sigue teniendo un peso importante y ya se conocen diversas experiencias en cuanto a formulaciones concretas en tal sentido. Pero por otro lado, surgen dudas acerca de su valor y posibilidades ya que, como todo proceso de cambio, puede quedarse en un nivel formal en el que sólo se convierta en un conjunto de formulaciones que elaboran un grupo de especialistas sin que estos cambios sean deseados, comprendidos y puestos en marcha por los actores curriculares decisivos como lo son los profesores en el marco de las instituciones en las que desarrollan su labor.

Desde este lugar nos preguntamos si el cambio curricular basado en competencias es percibido solamente como respuesta a demandas que provienen del exterior del campo educativo o si puede vivirse además como un cambio deseado y necesario o conveniente para mejorar la calidad de los profesionales que se forman en la universidad.

\section{Surgimiento y aclaraciones acerca de la noción de competencias}

Existe acuerdo entre los diversos autores que se han preocupado por el tema de las competencias, en cuanto a la ambigüedad y labilidad de este concepto, lo que precisamente dificulta su tratamiento y más aún, su uso en el campo educativo y curricular.

Si nos remontamos a su origen debemos reconocer que el movimiento moderno de las competencias proviene del campo de los negocios con los cambios acaecidos entre los 60 y los 70 (Mastache, 2007, p. 23). Desde el punto de vista académico debemos recurrir al ámbito de la lingüística y allí nos encontramos con que Chomsky, en 1964, construyó el concepto de "competencia lingüística" como objeto de estudio de ese campo del saber, buscando dar identidad a un conjunto de saberes y sentando las bases para su construcción.

A partir de allí puede encontrarse un uso que linda entre lo abusivo aplicado a distintos ámbitos sin que medie una reflexión rigurosa acerca de su origen, sentido y posibilidades de uso pertinente (Díaz Barriga, 2006, p. 13). Podemos, como ejemplo, mencionar los siguientes usos: competencia ideológica (1970) competencia discursiva (1982) y en el campo específico de la educación: competencia investigativa (2000); competencia metodológica.

El uso exagerado y sin la rigurosidad necesaria en el campo del saber no hizo más que atentar contra la claridad y precisión de un concepto que ya 
tenía una carga importante de ambigüedad y que genera polémica en el ámbito educativo.

Si volvemos al mundo del trabajo, en el que aparece este tema con mayor claridad, no podemos dejar de ver su sentido utilitario, ya que se apoya fundamentalmente en el análisis de tareas propias de un técnico, a partir del cual es posible identificar habilidades y destrezas para su desempeño en el trabajo de manera eficaz (Mastache, 2007) (3).

Pueden resultar aquí valiosos algunos análisis acerca del origen del concepto en el ámbito de las empresas y los negocios. El fordismo y el taylorismo (fines del siglo XIX hasta la década de los 70) fueron teorías que permitieron una planificación de los procesos productivos a partir de tener en cuenta los principios de división técnica y social del trabajo, lo que conduce a la distribución de funciones y de tareas entre individuos y grupos, no sólo laborales sino también sociales, desde las más simples a las que exigen mayor cualificación.

Para esta lógica de pensamiento y de organización del trabajo el análisis de las exigencias de cada puesto de trabajo con la finalidad de lograr mejor rendimiento, se convierte en el tema central. Sin embargo no todo se tradujo en progreso constante y mejor rendimiento. Comenzaron a surgir conflictos entre descripciones anímicas de acciones y tareas realmente realizadas. Está claro que ningún análisis y formulación teórica que solamente atienda a algunos aspectos de la cuestión de que se trate, puede explicar y orientar la práctica consecuente.

Otros autores como Elton Mayo y Mc Gregor, comienzan a preocuparse por otro aspecto del mundo del trabajo como lo es el de las relaciones humanas, la motivación, el liderazgo. Estos nuevos aportes ponen más en evidencia las limitaciones del modelo de organización del trabajo basado en principios de división técnica y social. De éste comienzan a surgir nuevos planteos encaminados a sistemas de producción más flexibles, que permitan atender a nuevos cambios que cada vez se producían con mayor rapidez y a la diversidad en las demandas. Agregado a lo anterior no podemos dejar de considerar los aportes de la tecnología en general y de la tecnología de la información, cuestiones que han contribuido a la celeridad de los cambios en los ámbitos del trabajo.

Ya es otro el tipo o los tipos de trabajadores y especialistas que se necesitan en el ámbito laboral, empresarial. Se requieren trabajadores con capacidades de integración y trabajo grupal, facilidad para el análisis y la solución de problemas, con flexibilidad para los cambios ante situaciones nuevas o diferentes a las conocidas. Y en consecuencia esto deriva en un nuevo problema para la educación: ¿cómo formar a este tipo de técnico y especialista requerido?, ¿cómo diseñar el curriculum y cómo concretar la enseñanza para facilitar estos cambios? Es así como surgen diferentes propuestas y se pone en discusión el ya conocido problema referido a qué y cuánta formación general habrá que ofrecer y cómo diseñar y desarrollar formaciones específicas, buscando tam- 
bién el desarrollo de capacidades referidas a la persona, y otros atributos más propios del ámbito de las relaciones sociales.

\section{Desplazamiento del concepto de "competencia" al campo educativo}

Si bien es cierto que en un principio el concepto tuvo mayor uso en el ámbito de la educación técnica sin causar demasiadas preocupaciones y debates acerca de su origen, significado y uso, la cuestión cobra otras dimensiones cuando se pretende extender a otros ámbitos como el de la Educación en general y especialmente en la Educación Superior.

Consideramos importante profundizar el debate en torno a esta cuestión del curriculum y la enseñanza atendiendo a un enfoque de competencias, en tanto el camino a su utilización en educación aparece cada vez más transitado, ayudando a ello algunas demandas que surgen ante este mundo cada vez más globalizado y la conformación de bloques o grupos económicos, políticos, culturales de distintos países.

En América Latina, si bien la incorporación del enfoque de competencias estuvo vinculado a las políticas de empleo (Mastache, 2007) hoy ya existen avances para establecer un Convenio Regional para la Convalidación de Estudios, Títulos y Diplomas de Educación Superior en América Latina y el Caribe.

Es conocido, también, que en el bloque del MERCOSUR se están buscando acuerdos globales de integración regional, referidos a la certificación de competencias en diversos campos y profesiones, lo que permitiría la libre circulación de personas con acreditación de los estudios realizados.

Ahora bien, si se pretende formar a través de la educación sujetos, profesionales, trabajadores, ciudadanos "competentes", ¿estaremos de acuerdo en lo que significa ser competente en algún campo o actividad determinada?, ¿cómo saber cuándo un sujeto es competente?, ¿cómo puede encarar esta cuestión la educación? Los interrogantes son muchos y pueden seguir sumándose. Anahí Mastache (2007), luego de analizar diferentes conceptualizaciones de competencias, extrae una serie de elementos comunes relacionados con aspectos centrales de las competencias, que la llevan a decir que las competencias:

- $\quad$ son propias de los sujetos considerados individualmente;

- la situación o contexto de la acción suele ser definido por su complejidad;

- $\quad$ permiten el logro eficaz de ciertos objetivos o niveles preestablecidos;

- $\quad$ se definen por resultado más que por la acción misma;

- $\quad$ son observables en el desempeño (resolución de un problema, toma de decisiones, etcétera);

- integran conocimientos, habilidades, destrezas, aptitudes; o el saber, el saber hacer y el saber ser; 
- $\quad$ el énfasis está en la habilidad más que en el conocimiento;

- algunas definiciones, aunque no todas, señalan como componente de la competencia la posibilidad de autonomía y flexibilidad en el desempeño de la tarea;

- $\quad$ en cuanto a la transferibilidad, Mertens (1996) reconoce la existencia de un debate respecto de si debe ser parte de la definición de competencia o si es un atributo a ser considerado a posteriori;

- $\quad$ en algunas definiciones las competencias se relacionan con el desempeño real correspondiente a un determinado empleo o medio laboral, son "situadas", contextuales; otras definiciones, por el contrario aluden a capacidades potenciales que pueden ser aplicadas en distintos contextos.

El análisis pormenorizado de los puntos anteriores permite visualizar una importante cantidad de temas y problemas que deben enfrentarse en el campo educativo si es que se pretende usar este concepto de competencias con solvencia y fundamentación. En principio, conviene aquí hacer algunas aclaraciones con respecto a las relaciones de las competencias con otra noción muy usada en educación como lo es la de capacidades. Buscando puntos en común entre diferentes conceptualizaciones es posible afirmar que la capacidad es un atributo de orden psico-cognitivo de los sujetos, que depende de la aptitud pero también del aprendizaje y de la experiencia previa. Representa la posibilidad de tener éxito en una tarea, en una profesión, etc. Algunos autores (Catalano, Avolio de Cols y Sladogma, 2004) sostienen que el desarrollo de capacidades es la base del despliegue y crecimiento de las habilidades o competencias.

Desde un punto de vista sociológico, Marcos Díaz Villa (2006, pp. 1-2) plantea que las competencias se construyen socialmente, que constituyen una realidad estructurante y estructurada, que le permite al sujeto desenvolverse en distintos planos, contextos y situaciones. Sostiene que, además de las condiciones subjetivas (muchas de ellas ya mencionadas), requieren de condiciones objetivas, produciéndose su desarrollo en el entrecruzamiento de ambas. Nos parece importante destacar entre las condiciones objetivas (4) y subjetivas que menciona este autor:

a. La competencia -como una construcción social- no puede abstraerse de los procesos y relaciones que la construyen, jerarquizan y legitiman.

b. La competencia se desarrolla y valida en instituciones especializadas cuyas ideologías y proyectos formativos están delineados y expresados en currículos y pedagogías específicas.

c. Dichos procesos formativos se realizan en el interior de campos de conocimiento y de práctica fundamentados en principios, teorías, hipótesis, interpretaciones, explicaciones y argumentos, que se comparten y que se consideran legítimos. En este sentido, el conocimiento profesional es un producto colectivo, social que, definido como legítimo, comparten los profesionales de una comunidad epistémica. 
d. Se configuran en prácticas de interacción (enseñanza-aprendizaje) en contextos específicos donde se refuerza la actividad y creatividad del estudiante, en relación con los saberes y prácticas propios del campo.

e. Su logro y desarrollo está asociado a fines y objetivos relacionados con el saber y el hacer dentro de contextos específicos (o, de manera transversal, a la relación entre contextos), y a criterios de actuación o desempeño verificables y demostrables.

Subjetivas porque:

a. La competencia se asocia de manera intrínseca a los sujetos, quienes la poseen y desarrollan (5).

b. El conocimiento es un substratum básico de la competencia, que acontece en la mente del hombre (Villoro, 1982).

c. La competencia se asocia a la manera como el sujeto se relaciona con objetos de conocimiento o aprendizaje.

d. Presupone ciertas facilidades intrínsecas a todos los individuos, que pueden considerarse semióticamente como una "gramática interna".

e. Se relaciona con rasgos y factores subjetivos de los individuos, en nuestro caso los futuros profesionales.

En principio podríamos afirmar con Díaz Barriga que, aunque no es fácil aceptar una conceptualización del término competencias, ellas suponen tres elementos: a) una información; b) el desarrollo de una habilidad y, c) puestos en acción en una situación inédita. La combinación de estos tres aspectos será entonces necesario tener en cuenta cuando pretendemos pensar en el concepto de competencias y su utilización en el campo educativo. Queda con ello claro que habrá necesariamente que superar la concepción enciclopedista de la educación, la concepción contenidista del curriculum para atender además de los contenidos, al desarrollo de habilidades y poder ofrecer contextos (reales o simulados) en los cuales los estudiantes puedan poner en movimiento sus competencias. Éste no es un desafío sencillo para las prácticas educativas, más allá de que parezca aceptarse el discurso que contemple lo anteriormente planteado.

La inclusión del concepto de competencias en el ámbito educativo ha producido y está produciendo importantes debates con posiciones diferentes que dan lugar a nuevas dudas e incertidumbres, sin embargo, el concepto está lejos de dejar de utilizarse. Podemos mencionar la posición de Short (citado en Carvajal Ruiz, 2001) (6) quien formula la crítica a la incorporación del término de competencia en el mundo de la educación, al sostener que el discurso y el vocabulario asociados al término es producto del deslizamiento de prácticas teóricas y experiencias derivadas de los aportes conductistas a la formación y gestión de personal en las empresas. Esta posición ha sido también adoptada 
por sectores de docentes y expertos que muestran resistencia a la incorporación en el ámbito educativo del concepto de competencias.

Cullen (1997, p. 90), en su obra Crítica de las razones de educar, al abordar el problema de la formación docente, entre otras cuestiones, se pregunta qué son las competencias y al hacerlo reconoce que todos los intentos de cambio o reforma curricular buscan formular las competencias necesarias para satisfacer las demandas sociales de aprendizajes. Plantea que en términos de historia curricular la noción de competencia es una categoría que reemplaza, y agrega, fuertemente a la de "objetivos educacionales" que tuvo preponderancia absoluta en la década de los 70 . Numerosos estudios y trabajos han desarrollado ampliamente este modelo al que Gimeno Sacristán lo caracteriza como una obsesión por la eficiencia (1985).

Es posible apreciar que las propuestas de desarrollo de las competencias varían de acuerdo a las concepciones que se sustenten respecto de cuestiones de base como las filosóficas-epistemológicas, psicológicas, pedagógicodidácticas.

Nosotros nos inclinamos por partir de la noción de competencia que propone Perrenoud (2002, p.7) (7) como "capacidad de actuar de manera eficaz en un tipo definido de situación, capacidad que se apoya en conocimientos, pero no se reduce a ellos". De este modo reconocemos que la educación no puede lograr sus intencionalidades ni satisfacer las demandas de distinto tipo que a ella se le formulan, si no avanza más allá de la transmisión de conocimientos y de cultura, para formar sujetos capaces de actuar adecuadamente ante las situaciones que le tocan vivir en el mundo social y profesional y los problemas que para ello debe resolver.

Uno de los aspectos más importantes de la inclusión de esta noción en el campo educativo es que significa un cambio de paradigma a la hora de plantear el problema de los objetivos y el modelo de enseñanza, pues se pasa de un enfoque centrado en el profesor a un enfoque centrado en el alumno y su proceso de aprendizaje. Esto implica otra manera de pensar y de desarrollar las actividades en la clase con un cambio en los papeles que desempeñan alumnos y docentes, priorizando el aprender a aprender en situación (en este sentido se puede mencionar como antecedente en educación el movimiento de la escuela activa). Este planteo remite, según Perrenoud, a un debate entre los paradigmas de la didáctica, afirmando por ejemplo, que este enfoque permite materializar planteos piagetianos con la conformación de "esquemas de acción". La enseñanza en base a problemas constituye una modalidad acorde con este enfoque, aunque su utilización en el desarrollo del curriculum merece una serie de consideraciones especiales. Por el momento podemos decir que en realidad el enfoque por competencias (Díaz Barriga, 2006) constituye una lucha contra el enciclopedismo en educación y entonces merece que se le preste atención. 


\section{El enfoque de competencias en el campo del curriculum en la educación superior}

No hay dudas en cuanto a que al nivel superior del sistema educativo le corresponde la formación de profesionales. Esta afirmación da lugar a especificidades en cuanto a la concepción, diseño y desarrollo del curriculum y, en el caso que estamos tratando acerca de la inclusión del concepto de competencias, el problema también presenta particularidades que trataremos de dilucidar.

En el caso de la educación superior, Díaz Villa (2006) sostiene que "el discurso constitutivo de las competencias especializadas ha tenido, en diferentes dimensiones y sentidos, una justificación basada en las necesidades de la sociedad y en la solución de los problemas que demanda, en ésta, el desarrollo. Así, mientras la visión tradicional de formación profesional planteaba como requisito para el ejercicio profesional la posesión de un cuerpo de conocimientos que se aplicaba a situaciones conocidas para producir una solución racional a los problemas, la visión actual -influenciada por los cambios producidos en la sociedad denominada post-industrial, de la información, o del conocimiento- (Castells, Gibbons, Lyotard) considera que la formación profesional debe responder a los retos que plantean las nuevas condiciones del ejercicio laboral, ya no dependiente de situaciones conocidas y rutinarias, sino de situaciones contingentes que demandan respuestas inteligentes a problemas nuevos y desconocidos en el nuevo mercado de trabajo. Esto significa que la formación ya no descansa en la transmisión de saberes predefinidos, sino que estos -saberes- son una función de la contingencia del mercado laboral. Se ha producido, de esta manera, una transformación en el discurso pedagógico que ahora genera nuevos órdenes de instrucción y nuevas formas de identidad (Bernstein, 1998)".

Consideramos pertinente analizar aquí las diferencias que J.M. Barbier (1999) (8) plantea entre tres mundos: profesión, formación y enseñanza, los que trabajan sobre hipótesis distintas y mantienen diferencias entre ellos. En el mundo de la profesión las competencias ocupan un lugar central, en tanto a través del desempeño profesional es donde se aprecian realmente las competencias en acción y movimiento ante los diferentes contextos en que el sujeto debe actuar. De allí que, en la formación profesional se trata de formar a través del trabajo mismo, entrenando y ejercitando al aprendiz en determinadas competencias.

El mundo de la enseñanza le corresponde a la escuela y a ella le corresponde, básicamente, la tarea de transmitir y comunicar el conocimiento a alumnos que tratan de apropiarlo. Éstas son las funciones que más conocemos. Pero a la educación también se le pide que cumpla con la función de formación, la que va más allá de la transmisión/apropiación del conocimiento. Se le pide que el alumno no sólo logre saber sino también saber hacer y saber ser, para ello será necesario ofrecerle situaciones en las que tengan que poner en juego lo 
aprendido en la formación. Y para que haya formación, según Barbier (1999), tiene que producirse la transferencia. Un claro ejemplo de formación sería lo que se espera que se produzca con la formación docente, en la universidad, en las escuelas técnicas, tarea que no concluye con la formación de grado sino que se profundiza y diversifica en estudios posteriores.

En el caso del curriculum en la universidad y en el nivel superior en general, el curriculum debe orientarse claramente a una formación profesionalizadora, mientras que la tendencia actual es la de dejar la especialización para el posgrado. Sin embargo queremos dejar en claro que nuestra posición respecto de la determinación del perfil profesional no es la de su construcción a partir solamente de las demandas del mundo laboral, sino que la universidad, por sus mismas funciones, tiene la obligación de producir una relectura de ellas y tener la autonomía para fijar competencias que tengan que ver con la ciudadanía y con necesidades sociales y políticas propias de diferentes contextos.

Ahora bien, ¿cómo concebimos al curriculum? Y aquí podemos encontrarnos también con ambigüedades y diferencias en el modo de conceptualizar este objeto complejo, lugar de confluencia de diferentes aspectos y problemas educativos. Nosotros sostenemos que el curriculum es un proyecto políticopedagógico, síntesis de posiciones filosóficas, sociológicas, epistemológicas, psicológicas, pedagógicas, históricas, y socialmente contextualizado, que expresa la intencionalidad de producir una intervención social planificada.

Sin embargo, la mirada a las políticas y las prácticas educativas actuales alertan en cuanto a la coexistencia de concepciones diversas, contrapuestas y/o contradictorias, que ponen de manifiesto que el curriculum es en realidad un campo de lucha y de confrontación de posiciones ideológicas, políticas, culturales, epistemológicas, pedagógicas puestas en juego a través de diferentes grupos de poder interesados en la educación. Este planteo no siempre es asumido de forma consciente por los docentes, lo que dificulta la comprensión por parte de ellos, de los complejos entrecruzamientos que se producen entorno al curriculum.

Desde el enfoque por competencias se argumenta que éste es de utilidad para concebir, diseñar y desarrollar el curriculum en el nivel superior dado que los perfiles académicos y profesionales se formulan a partir de la descripción de las competencias requeridas en el mundo de la profesión; dichos perfiles permiten la selección de contenidos en relación con ellos. Las competencias están formadas por una combinación dinámica de cualidades (Bolívar, 2007), que tienen que ver con el conocimiento, su aplicación y actitudes y responsabilidades ante el aprender y aprender a hacer. Así concebidas permiten la formulación de objetivos en términos de los que se espera que el alumno logre.

Resulta oportuno aquí hacer referencia al proyecto Tuning Educational Structures in Europe (9), que se propone la armonización de estudios superiores entre los países europeos y ha servido de modelo-base para el diseño 
de titulaciones, teniendo en cuenta el proceso de convergencia de Bologna (citado en Bolívar, 2007).

El modelo-base de referencia propone dos tipos de objetivos educativos que pueden ser definidos en términos de competencias:

- Competencias generales (transferibles y comunes a cualquier titulación) subdivididas en competencias instrumentales, interpersonales y sistémicas. Se refieren a cuestiones tales como capacidad de análisis, la cultura general, capacidad para trabajo autónomo, colaboración, organización, etc.

- Competencias específicas ligadas a las áreas de estudio (conocimiento teórico, práctico y/o experiencial y habilidades específicas del área). Tienen que ver con el manejo de métodos y técnicas propias de cada área de que se trate.

Más allá de las coincidencias o no que se puedan tener con estos planteos, existen algunos aspectos de la cuestión curricular, de orden más técnico, acerca de los que hay coincidencias; ellos tienen que ver con las respuestas que el curriculum tiene que dar a determinados interrogantes, como los siguientes:

- ¿Qué fines se desean alcanzar? El problema de los objetivos-competencias.

- ¿Qué deberán aprender los estudiantes y enseñar la institución educativa? El problema de los contenidos.

- ¿Qué experiencias y procesos de enseñanza y de aprendizaje ofrecen mejores posibilidades para alcanzar los objetivos? El problema de la metodología y las actividades.

- ¿ ¿Cómo se puede comprobar si se han alcanzado los objetivos o competencias propuestas? El problema de la evaluación.

- ¿Cómo organizar adecuadamente las experiencias y actividades de enseñanza y de aprendizaje? El problema de la temporalización.

En tanto la enseñanza es una actividad intencional que requiere ser diseñada la mirada experta a las planificaciones, o mejor proponemos la categoría de Proyecto de trabajo para cada espacio curricular, puede permitirnos tener indicios valiosos acerca de las concepciones curriculares y de la enseñanza y del aprendizaje, por parte de los profesores. Además de lo anteriormente planteado, es importante ver si en los proyectos de cátedra están claras cuestiones como las siguientes: ¿qué lugar ocupa la materia o espacio curricular a mi cargo dentro del curriculum de la carrera?, ¿cómo contribuye al logro de determinadas competencias?, ¿cómo se articula lo en ella aprendido con los otros aprendizajes que se realizan a través de la carrera?

En el caso del curriculum universitario es posible distinguir entre la necesidad de desarrollar conocimientos y habilidades (competencias) directamente relacionadas con las diferentes disciplinas, las que aportan aprendizajes básicos, y aquellas otras que requieren de procesos más complejos en los que están comprometidas más de una disciplina, es decir un conjunto de disciplinas. 
Aquí nos estaríamos refiriendo a competencias más generales y complejas que muchos autores coinciden en denominarlas como competencias transversales, las que implican aspectos complementarios que pueden ser utilizados en diferentes campos. Estas competencias pueden ser de dos tipos: aquellas relacionadas con el desempeño profesional y las vinculadas a ciertas actitudes, que por supuesto tienen que ver con determinados conocimientos pero además se relacionan con cuestiones de interés social amplio como, por ejemplo, los problemas ambientales. Este tipo de competencias pasa a ocupar un lugar central en la formación de profesionales ya que los problemas que tendrá que resolver en la vida profesional reclaman una conjunción de saberes y habilidades provenientes de distintos campos del saber (Díaz Barriga, 2006).

De todos modos, se trate de competencias disciplinares o de competencias transversales, lo que no se puede dejar de tener en cuenta es que para poner en juego las competencias es necesario producir la movilización de la información. Esta situación, según Perrenoud (2002), acerca este concepto de competencia al de "esquema de acción" de la teoría piagetiana, concepto que él retoma y desarrolla en el caso de la formación docente.

\section{La formación de competencias y la temporalidad. Alternativas en la construcción del curriculum en el nivel superior}

Una cuestión más a tener en cuenta en el enfoque de competencias es el de su logro procesual, es decir, a través del tiempo. Ello significa que una competencia no se logra en un solo momento o a través de una sola situación, requiere tiempo, el recorrido por el curriculum. Esto ha llevado a algunos autores como Roe (2003; citado en Díaz Barriga, 2006) a considerar que existe una etapa básica en la formación en competencias, que puede llamarse inicial y otra avanzada.

Del planteo anterior se deduce que la formación básica es también una formación en competencias profesionales, de allí que el curriculum y su desarrollo deben ofrecer oportunidades de integrar conocimientos y competencias y tienen que poner a los alumnos en situaciones de movilización de las competencias, y no dejar que esto lo hagan ellos solos y con posterioridad. Inevitablemente, entonces deben cambiar las clases en el nivel superior. No más enciclopedismo, pasividad, desintegración de contenidos y de situaciones de aprendizaje.

Pero aún tenemos que avanzar en otra cuestión problemática acerca de la elaboración de diseños curriculares desde esta perspectiva de competencias. Según Díaz Barriga (2006), existen dos estrategias diferentes: una que denomina enfoque integral por competencias, que recurre a distintas clasificaciones de competencias y se busca la demostración de competencias (generales o clave, derivadas, secundarias o particulares); busca permanentemente la solución de problemas y permite elaborar una visión integrada de la formación profesional de la educación superior. 
La otra estrategia metodológica del enfoque por competencias, que el autor denomina mixta, reconoce que hay momentos de aprendizaje de una disciplina que tiene que ver con la formación general del sujeto que hace a comprensiones de tipo más general que no requiere de la utilización directa del contenido aprendido. En realidad se trata de una metodología que combina el enfoque por competencias con otro como es el de contenidos. Esta estrategia es la que tiene que ver con la ya conocida formación en dos tramos: uno de formación básica (ciencias básicas) que tiene relación directa con los conocimientos propios de cada disciplina y otro de formación aplicada, centrada en el planteo de situaciones y problemas profesionales reales, con sistemas de pasantías y residencias. Para Díaz Barriga (2006), este último enfoque es más prometedor para incorporar las competencias en el plano curricular.

De todos modos lo que importa analizar en el curriculum es su coherencia interna y equilibrio entre los diferentes componentes ineludibles como lo son los Objetivos, los Contenidos, la Metodología y las Actividades y la Evaluación. Por otra parte será necesario ver las interpretaciones que de esos componentes realizan los profesores en sus diferentes proyectos de desarrollo y enseñanza para los distintos espacios curriculares.

\section{A modo de conclusiones}

En primer lugar insistimos en la importancia de debatir el tema en profundidad entre los diversos actores del diseño y desarrollo del curriculum que permita la clarificación del concepto y de sus usos. Planteamos, coincidiendo aquí con Bolívar (2007), que el debate sobre el nuevo diseño de titulaciones en términos de competencias no es sólo técnico, sino que por el contrario, es político ideológico, en tanto tiene que ver con la función de la universidad en relación con la profesionalidad y la formación.

Uno de los problemas o riesgos, desde nuestro punto de vista, que puede tener la interpretación y diseño del curriculum siguiendo el enfoque de competencias, es que aquellas más generales, con intenciones y posibilidades integradoras del curriculum, comiencen a ser discriminadas en competencias más específicas y simples, con indicadores puntuales, que termine convirtiéndose nuevamente en un modelo por objetivos, en el que cada competencia simple coincide con los objetivos específicos formulados. Ésta es una de nuestras hipótesis respecto del curriculum universitario, si es que no se trabajan previa y simultáneamente las concepciones epistemológicas, psicológicas, políticosociales y pedagógicas que hacen a los fundamentos del curriculum.

Otro riesgo puede ser el interpretar la organización del diseño curricular total, en términos de traducción de las competencias a situaciones problemáticas, lo que puede significar que los contenidos queden supeditados a las necesidades que las diferentes situaciones problemáticas requieran, perdién-

dose así la concepción más integral de los conocimientos y de las disciplinas 
a las que pertenecen, no permitiendo de este modo apropiarse de la estructura interna de cada una de ellas.

Podemos afirmar, coincidiendo con Díaz Barriga (2007), que no existe hasta el momento una propuesta clara y definitiva acerca del empleo del enfoque por competencias en el campo de la educación y del curriculum, en el que el exceso de clasificaciones de competencias no hace sino oscurecer su utilización.

Lo anterior nos permite concluir que es difícil construir una metodología de diseño curricular apoyada en el enfoque por competencias, sin embargo consideramos que puede ofrecer aportes a la tarea de diseño y más aún en el desarrollo del curriculum, dado que lo que realmente tendría que cambiar, si queremos formar profesionales y ciudadanos competentes, es, precisamente, lo que ocurre en las aulas, en los modelos de enseñanza y en las instituciones formadoras.

Debemos tener en claro que el sistema educativo y la formación profesional responden a lógicas diferentes tal como lo plantea A. Mastache (2007). La educación formal aporta sólo una parte de la formación profesional requerida en el desempeño laboral. Las competencias requieren de una buena base que puede aportar la educación formal pero será el mundo del trabajo el que pondrá a los sujetos ante situaciones reales que requerirán de las competencias puestas en juego y en situación. Los perfiles de competencias requeridos en el mundo laboral constituyen un insumo valioso para diseñar un curriculum que acerque más al estudiante a su futuro espacio laboral. Por ello decimos que el perfil del egresado, aspecto tan importante del curriculum del nivel superior, no puede surgir solamente ni linealmente de las competencias profesionales identificadas.

\section{En búsqueda de criterios básicos para abordar el cambio del curriculum}

Desde la perspectiva de políticas curriculares y de organización institucional, el problema trasciende lo didáctico. Entonces, el curriculum puede ser considerado como un proceso de toma de decisiones, cuyo sentido, coherencia y sistematización, hacen a su vez que el análisis desde la didáctica, la administración y la organización educativa, converjan en el plano de la política de la educación.

El curriculum universitario considerado entonces como proyecto políticopedagógico y como proceso de toma de decisiones requiere también de distintos niveles de análisis. Tal como ya se dijera, desde la política educativa resultan pertinentes tanto el análisis macro como micropolítico. Desde la mirada macropolítica, habrá que indagar acerca de las políticas más generales y de su concreción en normativas que tiendan a configurar un marco legal general, que atienda al principio de centralización y de homogeneización, a la vez que genere 
espacios para la descentralización y la autonomía necesaria para atender a los problemas de diversidad, sosteniendo la calidad.

Siguiendo en esta línea de análisis, en primer lugar, consideramos indispensable crear las condiciones y espacios de trabajo y de debate en los ámbitos institucionales que permitan iniciar procesos de reflexión y de diseño de políticas tendientes a producir los cambios curriculares que, tanto los nuevos aportes teóricos como el análisis de las prácticas actuales, como así también las nuevas condiciones sociales reclaman. Esta tarea puede ser una responsabilidad de las autoridades de gestión universitaria o bien planteada como demanda y derecho de profesores y estudiantes. En cualquiera de los casos es importante trabajar en el sentido de la búsqueda de criterios básicos para abordar en el cambio. En tal sentido se enuncian a continuación una serie de cuestiones a tener en cuenta como punto de partida.

1) Los cambios curriculares son necesarios pero no suficientes para producir la transformación deseable para la universidad.

2) Como paso inicial es necesario realizar un diagnóstico de situación local pero ubicándolo en el contexto regional, nacional y mundial.

3) Tener presente que los campos de interés de la universidad son los sistemas productivos de bienes, servicios y conocimientos.

4) La pertinencia de mantener una perspectiva amplia, abierta y flexible frente a:

- La existencia de nuevos campos de conocimientos.

- El surgimiento de nuevos problemas científicos.

- La aparición de nuevas áreas profesionales y la transformación o desaparición de otras.

- Los nuevos requerimientos en cuanto a las competencias profesionales en relación a nuevas formas de trabajo y de vida.

- La necesidad de comprensión de los problemas de la sociedad actual y de implicancias ético-sociales del ejercicio profesional.

5) El reconocimiento de que la universidad no forma sólo profesionales sino que también tiene fundamental importancia en la formación de concepciones del mundo, de la vida, del desarrollo social e individual y en los modos de organización social y de participación política.

6) Tener en cuenta que la formación académica debe estar dirigida al desarrollo y apropiación crítica de conocimientos teóricos, técnicos, procedimentales y actitudinales o de competencias.

7) Los contenidos como las prácticas educativas tienen que posibilitar tanto la autonomía personal (en el pensamiento, en la toma de decisiones, en el manejo de la información) como una formación versátil que le permita al sujeto enfrentar la incertidumbre y los cambios que se producen en la sociedad y en las teorías y paradigmas científicos. 
De acuerdo con lo hasta aquí expuesto, y siguiendo nuestra línea de trabajo, proponemos concebir al curriculum como el Proyecto educativo de la universidad, síntesis de posiciones político, sociales, epistemológicas, pedagógico-didácticas y profesionales, que ofrezca la flexibilidad suficiente como para ser revisado y reestructurado periódicamente para atender al rápido crecimiento del conocimiento y a las nuevas demandas sociales.

\section{Notas Bibliográficas}

(1) Bolívar, A. (2007) La planificación por competencias en la reforma de Bologna para la educación Superior. Un análisis crítico. Dossier.

(2) Díaz Barriga, A. (2006) El enfoque de competencias en educación ¿Una alternativa o un disfraz de cambio?. Revista Perfiles Educativos.

(3) Mastache, A. (2007) Formar personas competentes. Desarrollo de competencias tecnológicas y psicosociales. Buenos Aires, Noveduc.

(4) Díaz Villa, M. (2006) Hacia una sociología de las competencias. Santiago de Cali, Colombia.

(5) Para algunos enfoques, la competencia está asociada al logro de objetivos específicos (cognitivos, afectivos, psicomotores e interpersonales).

(6) Carvajal Ruiz, S. (2001) "Las competencias: concepto, definiciones, usos y aplicaciones". Revista Alternativas, LAE, Año VI, Nº 25, San Luis, Argentina.

(7) Perrenoud, P. (2002) "Construir competencias en la escuela" (2a . Ed.). Santiago de Chile, Dolmen, p. 125.

(8) Barbier, J. M. (1999) Prácticas de formación, evaluación y análisis. Buenos Aires, Novedades Educativas.

(9) Recientemente (2006) se ha publicado el informe final Tuning fase 2. La web del Proyecto puede verse en la Universidad de Deusto: http://www.unideusto. org./tuning subdividida en Europa y América Latina.

\section{Referencias Bibliográficas}

- $\quad$ Aristimundo, A. (2005) "Las competencias en la educación superior: ¿ demonio u oportunidad?". Departamento de Educación, Universidad Católica del Uruguay. En: www.upf.edu/bolonya/butlletins/2005/febrero1/demonio.pdf. Consultado el 06 de mayo de 2005.

- Barbier, J.M. (1999) Prácticas de formación, Evaluación y análisis. Buenos Aires, Novedades Educativas.

- Bolívar, A. (2007) "La planificación por competencias en la reforma de Bologna de la educación superior. Análisis crítico". Dossier EDT-Educacao Temática Digital, Campinas, V. 9, N Esp., pp. 68-94, ISSN 1676-2592.

- $\quad$ Carvajal Ruiz, S. (2001) "Las competencias: concepto, definiciones, usos aplicaciones", Revista Alternativas, LAE, Año VI, № 25, San Luis, Argentina. 
- Catalano, A., Avolio de Cols, S. y Sladogma, M. (2004) Competencia laboral. Diseño curricular basado en normas de competencia laboral. Conceptos y orientaciones metodológicas. Buenos Aires, CINTERFOR/OIT, p. 225.

- Cullen, C. (1997) Crítica a las razones de educar. Buenos Aires, Paidós.

- Diaz Barriga, A. (2006) "El enfoque de competencias en educación. ¿Una alternativa o un disfraz de cambio?", Perfiles educativos, V. 28, $\mathrm{N}^{\circ} 111, \mathrm{pp}$. 7-36, Disponible en Internet: http://redalyc.uaemex.mx/. Consultado el 14 de junio de 2007.

- Diaz Barriga Arceo, F. y Hernández Rojas, G. (2002) Estrategias docentes para el aprendizaje significativo. Una interpretación constructivista (2a ${ }^{\mathrm{a}}$. Ed.). México, Mc Graw Hill, p. 465.

- Diaz Villa, M. (2006) Hacia una Sociología de las competencia. Santiago de Cali, Colombia. Dossier.

- Comisión Europea (2003) "Las competencias clave. Un concepto en expansión dentro de la educación general obligatoria". Bruxelas, Eurydice, p.186. Disponible en Internet: http://www.eurydice.org/ressources/eurydice/ pdf/0_integral/032ES.pdf. Consultado el 24 junio de 2007.

- Mastache, A. (2007) Formar personas competentes. Desarrollo de competencias tecnológicas y psicosociales. Buenos Aires, Novoeduc.

- Perrenoud, P. (2002) "Construir competencias en la escuela” (2a . Ed.). Santiago de Chile, Dolmen, p. 125. y otros (Coords.) (2005) La formación profesional del maestro. Estrategias y competencias. México, Fondo de Cultura Económica. 